\section{Signal Recovery from Wavelet Transform Maxima}

\author{
A. Enis Çetin and Rashid Ansari
}

Abstract-This paper presents an iterative algorithm for signal recovery from discrete-time wavelet transform maxima. The signal recovery algorithm is developed by using the method of projections onto convex sets. Convergence of the algorithm is assured.

\section{INTRODUCTION}

Multiresolution representation of signals finds applications in several fields including computer vision, image processing, and geophysics. Recently, it is shown in [1] that the wavelet orthonormal bases [2]-[6] serve to provide a useful multiresolution signal representation, and some applications of the Wavelet Transform (WT) to signal and image analysis are presented. In [5], [6] it is observed that the local maxima of the absolute value (henceforth referred to as absolute maxima) of the WT of a signal help detect sharp variations in the signal, and an iterative signal recovery algorithm is developed from this information. However, the proof of convergence is not established and it is posed as an open problem.

This note presents an iterative algorithm for signal recovery from Discrete-time WT (DWT) absolute maxima. The new signal recovery algorithm is developed by using the method of the Projections onto Convex Sets (POCS) [7]. The key contribution here is that sets which are different from those used in [5], [6] are defined to represent the available information. The nature of these sets leads to establishing the convergence of the new algorithm for recovery problems that involve either 1) all absolute extrema, 2) absolute maxima, 3) extrema, or 4) maxima, of the DWT.

\section{Wavelet Transform and the Signal Recovery Problem}

In this section, the signal recovery problem is presented.

Let $z^{\prime}$ be a wavelet and $\phi$ be the corresponding smoothing function [1]. Let $\{d[n], n \in Z\}$ be a discrete-time signal that is to be recovered. It is assumed that $d[n]$ is acquired from a function $f \in L_{2}(R)$ such that $d[n]=\left.S_{1} f(x)\right|_{x=n}$ for all $n \in Z$ where $S_{1} f(x)=f * \phi(x)$. Alternately, by using a Lemma of [1], the existence of such a function $f \in L_{2}(R)$ can be assumed. The sequence $d[n]$ is therefore a uniformly sampled version of the function $f$ smoothed at resolution scale $2^{0}$ using the terminology of WT representation. The sequence $\left\{\left.S_{1} f(x)\right|_{x=n}\right\}$ is denoted by $S_{1}^{d} f[n]$. For a class of wavelets the following set of

$$
\left\{\left\{S_{2 L}^{d} f[n], n \in Z\right\} ; \quad\left\{W_{2^{l}}^{d} f[n], n \in Z . l=1.2, \cdots . L\right\}\right\}
$$

where $W_{2^{l}}^{d} f[n]=\left.f(x) * 2^{-l} \iota^{\prime}\left(2^{-l} x\right)\right|_{x-n}$ is called the Discretetime wavelet transform (DWT) of $d[n]$. The recursive computation of the DWT of $d[n]$ is now described. The sequences $S_{2^{l}}^{d} f[n]$ and $W_{2^{l}}^{d} f[n]$ are obtained from $S_{20}^{d} f[n]$ by convolving $S_{20}^{d^{2}} n[n]$ with digital filters $h$ and $g$ [4], respectively, i.e., $W_{2^{\prime}}^{d} f[n]=\left(S_{1}^{d} f * g\right)[n]$

Manuscript received November 30, 1991: revised February 11, 1993 The associate editor coordinating the review of this paper and approving i for publication was Prof. Monson Hayes. This work was supported in part by TUBITAK and in part by NATO.

A. E. Cetin is with the Department of Electrical Engineering, Bilkent University, Bilkent, 06533, Ankara, Turkey.

R. Ansari is with Bellcore, Morristown, NJ 07960

IEEE Log Number 9213283. and $S_{2^{\prime}}^{d} f[n]=\left(S_{1}^{d} f * h\right)[n]$ where the filters $h$ and $g$ are related to the wavelet $C$ and the smoothing function $o[1]$. In general,

$$
u_{2^{l+1}}^{d} f[n]=\left(S_{2^{l}}^{d} f * g_{l}\right)[n]
$$

and

$$
S_{2^{i+1}}^{d} f[n]=\left(s_{2^{i}}^{d} f * h_{l}\right)[n]
$$

where the digital filters $g_{l}$ and $h_{l}$ are obtained by inserting $2^{l}-1$ zeros between each coefficients of the filters $g$ and $h$. respectively. This implies that $H_{l}(\omega)=H\left(2^{l} \omega^{\prime}\right)$ and $G_{l}(\omega)=G\left(2^{l} \omega\right)$. In this way the DWT $\left\{\left\{S_{2 L}^{d} f[n]\right\}:\left\{W_{2^{\prime}}^{d} f[n] . l=1.2, \cdots, L\right\}\right\}$ are obtained from $d[n]$ in a tree-like structure.

An absolute maximum of the discrete-time signal $W_{2 l}^{d} f$ is defined as any sample $W_{2^{l}}^{d} f[n]$ satisfying the conditions (i) $\left|W_{2^{l}}^{d d} f[n]\right| \geq$ $\left|W_{2^{l}}^{-d} f[n-1]\right|$ and (ii) $\left|W_{2^{\prime}}^{d} f[n]\right| \geq\left|W_{2^{l}}^{d} f[n+1]\right|$. with the following qualifiers. If i) is satisfied with equality, then ii) should be satisfied with strict inequality along with the restriction that $\left|W_{2^{l}}^{d} f[n-1]\right|>$ $\left|W_{2^{l}}^{-d} f[n-2]\right|$. On the other hand if ii) is satisfied with equality, then i) should be satisfied with strict inequality along with the restriction that $\left|W_{2^{l}}^{d} f[n+1]\right|>\left|W_{2^{l}}^{d} f[n+2]\right|$. The DWT absolute maxima representation of a signal $d[n]$ is defined [5] as the absolute maxima of $W_{2^{l}} f . l=1.2, \cdots . L$. and the samples of the signal $S_{2^{l}}^{d} f$. The signal recovery problem is the reconstruction of $d[n]$ from its DWT absolute maxima representation.

\section{Iterative Signal Recovery Algorithm}

This section describes the new signal recovery algorithm which is devised by using the method of projections onto convex sets (POCS) [7].

The method of POCS has been successfully used in many signal recovery and restoration problems [8]. The key idea is to obtain a solution which is consistent with all the available information. In this method, the set of all possible signals is assumed to be a Hilbert space with an associated norm (in this note the Hilbert space is $t_{2}$ with Euclidian norm). All a priori information about the desired signal is modeled as convex sets, if possible. Let us suppose that the information about the desired signal is associated with $M$ sets, $C_{m}, m=1.2, \cdots, M$. Since, the desired signal satisfies all of the constraints it must be in the intersection set $C_{0}=\cap_{m=1}^{M} C_{m}$. Any member of the set $C_{0}$ is called a feasible solution [8]. If all of the sets $C_{m}$ are closed and convex then a feasible solution can be found by making successive orthogonal projections onto sets, $C_{m}$. Let $P_{m}$ be the orthogonal projection operator onto the set $C_{m}$. The iterates, $y_{k+1}=P_{1} P_{2} \ldots P_{11} y_{k}, k=0.1 . \cdots$ converge to a member of the set $C_{0}$. regardless of the initial signal $y_{0}$. If the solution is unique and $C_{m} . m=1.2, \cdots, M$, uniquely characterize the solution, then $C_{0}$ contains a single element (the solution) and the iterates converge to this element.

Let $W_{,,}^{-d} f\left[n_{k_{1}}\right] k_{l}=1.2 . \cdots . K_{i}$ be the DWT absolute maxima of the signal $d[n]$ at resolution scale $2^{l}$. Corresponding to each sample $\mathbb{U}_{2^{\prime}}^{d} f\left[n_{k_{l}}\right]$ a set $C_{2^{l}} k_{l}$ is defined in $\ell_{2}$. The set $C_{2^{l}} \cdot k_{l} \cdot\left(k_{l}=\right.$ $\left.1.2, \cdots . \Lambda_{l} ; l=1.2, \cdots, L\right)$ is the set of discrete-time signals $y \in l_{2}$ which have the DWT absolute maximum $W_{2^{l}}^{d} f\left[n_{k_{1}}\right]$ at the resolution scale $2^{l}$. i.e.,

$$
\begin{aligned}
C_{2^{l} \cdot k_{l}}=\left\{y:\left(y *^{i} g\right)\left[n_{k_{l}}\right]=W_{2^{l}}^{d l} f\left[n_{k_{l}}\right]\right\} . \\
\left(k_{1}=1.2, \cdots, K_{l}: l=1,2, \cdots, L\right)
\end{aligned}
$$

where the digital filter ${ }^{l} g$ is the inverse Fourier transform (FT) of ${ }^{l} G\left(\omega^{\prime}\right)=G\left(2^{l-1} \omega^{\prime}\right) \times \Pi_{i=0}^{i-2} H\left(2^{\prime} \omega^{\prime}\right)$. for $l>1$. and ${ }^{l} G\left(\omega^{\prime}\right)=G\left(\omega^{\prime}\right)$. 
The filter ${ }^{l} g$ is obtained by noting that $W_{2^{l}}^{d} f[n]$ is recursively constructed from $d[n]$ in $l$ stages. In the first stage, $S_{2 l}^{d} f[n]$ is obtained by convolving $d[n]$ with $h$. In the second stage $W_{2^{2}}^{d} f[n]\left(S_{2^{2}}^{d} f[n]\right)$ is obtained by convolving $S_{2^{l}}^{d} f[n]$ with $g_{2}\left(h_{2}\right)$, and so on.

Let $y_{1}$ and $y_{2}$ be any arbitrary members of $C_{2^{l}, k_{l}}$. The convex combination, $y_{t}=t y_{1}+(1-t) y_{2}, 0<t<1$, of $y_{1}$ and $y_{2}$ satisfies $\left(y_{t} *{ }^{l} g\right)\left[n_{k_{l}}\right]=W_{2^{l}}^{d} f\left[n_{k_{l}}\right]$ which means that $y_{l} \in C_{2^{l}, k_{l}}$, too. Therefore the set $C_{2^{l} \cdot k_{l}}$ is convex. The closure of $C_{2 l} l k_{l}$ can be established as in [7].

For any given sample $W_{2 l}^{d} f\left[n_{0}\right]$ of the signal $W_{2 l}^{d} f$ a set $C_{l, n_{0}}$ is defined as in (4), i.e.,

$$
C_{l, n_{o}}=\left\{y:(y * g)\left[n_{o}\right]=W_{2^{l}}^{d} f\left[n_{o}\right]\right\} .
$$

The set $C_{l, n_{0}}$ is also closed and convex in $\ell_{2}$. Therefore we can associate convex sets with the minima of the signals $W_{2 l}^{d} f, l=$ $1,2, \cdots, L$, as well.

Similarly, corresponding to the sample $S_{2 L}^{d} f\left[n_{m_{L}}\right]$ of the signal $S_{2 L}^{d} f, C_{S, m_{L}}\left(m_{L}=1,2, \cdots, M_{L}\right)$ is defined as the set of discretetime signals $y \in \ell_{2}$ which have the sample value $S_{2 L}^{d} f\left[n_{m_{L}}\right]$ at resolution $2^{L}$, i.e.,

$$
\begin{gathered}
C_{S, m_{L}}=\left\{y:\left(y *{ }^{L} h\right)\left[n_{m_{L}}\right]=S_{2^{l}}^{d} f\left[n_{m_{L}}\right]\right\} . \\
\left(m_{L}=1,2, \cdots, M_{L}\right)
\end{gathered}
$$

where the filter ${ }^{L} h$ is defined as the inverse FT of ${ }^{L} H(\omega)=$ $\Pi_{l=0}^{L-1} H\left(2^{l} \omega\right)$.

For any given WT absolute maximum $W_{2^{l}}^{d} f\left[n_{k_{l}}\right]$ one can define a set which assures local maximality as well. Let $C_{2^{l}, k_{l}}^{+}=\{y:(y *$ $\left.\left.{ }^{l} g\right)\left[n_{k_{l}}+1\right] \leq W_{2^{l}}^{d} f\left[n_{k_{l}}\right]\right\}$ and $C_{2^{l}, k_{l}}^{-}=\left\{y:\left(y *{ }^{l} g\right)\left[n_{k_{l}}-1\right] \leq\right.$ $\left.W_{2^{l}}^{d} f\left[n_{k_{l}}\right]\right\}$ with qualifiers similar to those mentioned in Section II. The sets $C_{2^{l}, k_{l}}^{+}$and $C_{2^{l}, k_{l}}^{-}$assure the local maximality of the value $W_{2^{l}}^{d} f\left[n_{k_{l}}\right]$ Actually, one can combine them and define a new set

$$
C_{2^{l}, k_{l}}^{*}=\left\{y:\left(y *{ }^{l} g\right)\left[n_{k_{l}} \pm 1\right] \leq W_{2^{l}}^{d} f\left[n_{k_{l}}\right]\right\} .
$$

All of the sets $C_{2^{l}, k_{l}}^{+}, C_{2^{l}, k}^{-}$and $C_{2^{l}, k_{l}}^{*}$ are closed and convex in $\ell_{2}$. Another convex set which can be used in the recovery algorithm is the bounded energy set, $C_{e}=\left\{y:\|y\|_{2}^{2} \leq \epsilon_{o}\right\}$ which is the set of sequences whose energy is bounded by $\epsilon_{o}$ [9], [10].

The proofs of closure and convexity of the above sets are omitted in this short paper.

The key operation of the method of POCS is the orthogonal projection onto a convex set. The projection, $y_{p}$. of any sequence $y_{o} \in \ell_{2}$ onto the set, $C_{2^{l}} \cdot k_{l}$, is the solution of the following constrained minimization problem

$$
\min \left\|y-y_{0}\right\|_{2}, \quad \text { subject to }\left(y *^{l} g\right)\left[n_{k_{l}}\right]=W_{2^{l}}^{d} f\left[n_{k_{l}}\right] .
$$

We obtain the solution of (8) by assuming that the filters $h$ and $g$ are FIR filters (therefore the filters ${ }^{\prime} g, l=1,2, \cdots, L$ and ${ }^{L} h$ are FIR filters as well). The projection vector $y_{p}=\left\{y_{p}[n]\right\}$ is given by

$$
\begin{aligned}
y_{p}[n] & =y_{o}[n]+\frac{W_{2^{l}}^{2} f\left[n_{k_{l}}\right]-\left({ }^{l} g * y_{o}\right)\left[n_{k_{l}}\right]^{l}}{\sum_{k}{ }^{l} g[k]^{2}} g\left[n_{k_{l}}-n\right] n \\
& =-p,-p+1, \cdots, 0,1,2, \cdots, q
\end{aligned}
$$

where we assumed that $y_{o}$ is a finite-extent sequence with $p+q+1$ possibly nonzero samples. Solution for IIR filters and infinite-extent sequences can also be obtained. In practice mostly FIR filters are used and the signals that we consider are finite-extent sequences or images [5], [6].

The projection $y_{p}$ of any sequence $y_{0} \in \ell_{2}\left(y_{0} \in C_{S, m_{L}}\right)$, onto the set $C_{S, m_{L}}$ can be obtained in a similar manner. The projection vector $y_{p}=\left\{y_{p}[n]\right\}$ is given by

$$
\begin{aligned}
y_{p}[n] & =y_{o}[n]+\frac{S_{2 L}^{d} f\left[m_{L}\right]-\left({ }^{L} h * y_{o}\right)\left[m_{L}\right]}{\sum_{k}{ }^{L} h[k]^{2}} h\left[m_{L}-n\right] n \\
& =-p,-p+1, \cdots, 0.1 .2, \cdots, q .
\end{aligned}
$$

Let us describe the signal recovery algorithm from WT maxima. The algorithm starts with an arbitrary initial estimate $y_{0} \in l^{2}$ which is successively projected onto the sets $C_{2^{l}, k_{l}}, k_{l}=1,2, \cdots, K_{l}, l=$ $1,2, \cdots, L$, and $C_{S, m_{L}}, m_{L}=1,2, \cdots, m_{L}$ by using (9) and (10). If the energy information is wished to be used then the current iterate is projected onto the set $C_{\epsilon}$, too. The order of projections is immaterial. In this manner the first iteration cycle is completed and the first iterate $y_{1}$ is obtained. The procedure is repeated until a satisfactory level of error difference in successive iterations is obtained.

If the WT minima information is available, then this information can also be used in the signal recovery algorithm because convex sets can be associated with the WT minima.

A simulation example is now described to show the use of the algorithm. Consider the cubic spline wavelet $\psi,[5]$ and the corresponding filters $\{h[n]\}=$ $\{\cdots, 0,0.625,0.25,0.375,0.25,0.0625,0, \cdots\}$, and $\{g[n]\}=$ $\{\cdots,-0.00008,0.0164,0.10872,0.59261,0,0.59261,0.10872$, $0.0164,0.00008, \cdots\}$ described in [5]. The digital filter $h[n]$ is an FIR filter and the coefficients of $g[n]$ decay exponentially (for all practical purposes $g[n]$ can be arbitrarily closely approximated by an FIR filter). Let $\{d[n]\}=$ $\{\cdots, 0,100,100,200,200,175,150,125,100,100,0, \cdots\} \quad$ be the test signal. The coarse signal $S_{2^{2}}^{d} f$, and the signals $W_{2^{l}}^{d} f$ and $W_{2^{2}}^{d} f$ are determined in order to generate the data for testing the algorithm. The signal recovery problem is the reconstruction of the signal $d[n]$ from $S_{2^{2}}^{d} f$, and the absolute maxima of the signals $W_{2^{l}}^{d} f$ and $W_{2^{2}}^{d} f$. An all zero sequence is selected as the initial estimate for the iterative algorithm, the choice for the initial estimate being arbitrary. In each iteration cycle, successive orthogonal projections onto the sets $C_{2^{1} . k_{1}}, k_{1}=1,2,3, C_{2^{2}, k_{2}}, k_{2}=1,2$, and $C_{S, m_{2}} m_{2}=1,2, \cdots, 22$ are performed using (9) and (10). The percent error $100 \times\left\|y_{k}-d\right\|^{2} /\|d\|^{2}$ where $y_{k}$ is the $k$ th iterate versus the number of iteration cycles is shown in Fig. 1. Clearly, we recover the original signal with negligible error.

In all the examples tried, a consistent behavior of the algorithm was observed.

The procedure can also be the basis of a scheme for information storage using the DWT maxima representation described earlier. In such a scheme the DWT maxima can be readily used for feature analysis, and when necessary, the signal information can be at least partially recovered using the iterative algorithm. With some variation the iterative algorithm is applicable to the cases that involve either 1) all absolute extrema, 2) absolute maxima, 3) extrema, or 4) maxima, of the DWT. Recently we became aware of two recent reports [11], [12] by Mallat $e t$ al. In these reports, a convergent iterative algorithm using different convex sets is developed for signal recovery from Continuous-time WT (CWT) maxima.

It was shown [12]-[14] that the local WT maxima do not uniquely characterize an arbitrary signal. However, it was experimentally observed in [12] that for most practical signals WT maxima provide a 


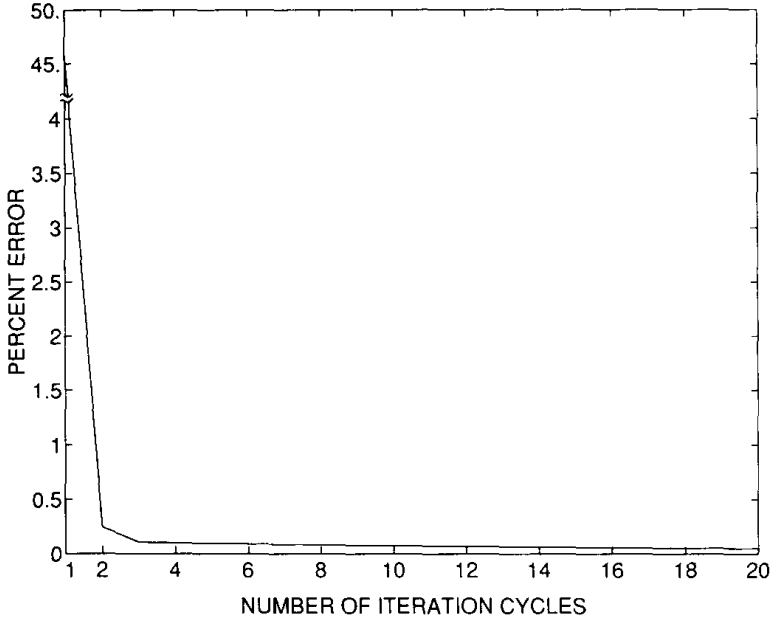

Fig. 1. Percent error versus the number of iterations cycles.

good representation of the original signal. We verified this observation in this paper. If there are multiple solutions of the signal recovery problem then our algorithm converges to one of the solutions because every solution is a member of the intersection set, $C_{o}$, as described in the beginning of this section.

The signal recovery technique can also be extended to multidimensional wavelets.

\section{REFERENCES}

[1] S. G. Mallat, "A theory for multiresolution signal decomposition: The wavelet representation," IEEE Trans. Patt. Anal. Mach. Intell., vol. 11, pp. $674-693,1989$

[2] Y. Meyer, Ondelettes et Operateurs. Paris: Hermann, 1988.

[3] J. Stromberg, "A modified Franklin system and higher order systems of $R^{n}$ as unconditional bases for Hardy spaces," Conf. in harmonic analysis in honor of $A$. Zygmund, Wadsworth Math. Series, vol. 2, pp. $475-493$.

[4] I. Daubechies, "Orthogonal bases of compactly supported wavelets," Commun. Pure Appl. Math., vol. XLI, pp. 909-996, 1988.

[5] S. G. Mallat and S. Zhong. "Complete signal representation with multiscale edges," Courant Inst., New York Univ., New York, Tech. Rep. 483, Dec. 1989.

[6] S. G. Mallat, "Zero-crossing of a wavelet transform," IEEE Trans. Informat. Theory, vol. 37, pp. 1019-1033, 1991.

[7] D. C. Youla and H. Webb, "Image restoration by the method of Convex Projections, Part 1-Theory," IEEE Trans. Med. Imaging, vol. 1, pp. $81-94,1982$.

[8] P. L. Combettes and M. R. Civanlar, "The foundations of set theoretic estimation," ICASSP'91, pp. 2921-2924.

[9] M. I. Sezan and H. Stark, "Image restoration by the method of convex projections, Part 2: Applications and numerical results," IEEE Trans. Med. Imaging, vol. 1, pp. 95-101, 1982.

[10] A. E. Çetin, "An iterative algorithm for signal reconstruction from bispectrum," IEEE Trans. Signal Processing, vol. 39, pp. 2621-2628, 1991.

[11] S. Mallat and S. Zhong, "Characterization of signals from multiscale edges," Courant Inst., New York Univ., New York, Tech. Rep. 592 Nov. 1991.

[12] S. Mallat and W. L. Hwang, "Singularity detection and processing with wavelets," IEEE Trans. Informat. Theory, vol. 38, pp. 617-643, Feb. 1992.
[13] Y. Meyer, "Un contre-example a la conjecture de Marr et a celle de S. Mallat," preprint, 1991.

[14] Z. Berman and J. S. Baras, "A study on discrete multiscale edge representations," in Conf. Informat. Sci. and Syst., Princeton Univ., Princeton, NJ, Mar. 1992.

\section{Inverting Periodic Filters}

Ching-An Lin and Chwan-Wen King

Abstract - We consider linear periodic filters. We give simple necessary and sufficient conditions for the filter to be invertible and a simple formula to compute its inverse. If the filter is not invertible, we propose a method to compute its optimal approximate inverse. An illustrative example is given.

\section{INTRODUCTION}

Periodic filters have been found useful in speech scrambling [3], in filtering of cyclostationary signals [1], and in decimator-interpolator filter design to reduce the required computations [7]. The inverse or an "approximate inverse" of a periodic filter is required to recover the scrambled signal [3]. Inverting a class of periodic filters is discussed in $[10]$.

We study the problem of finding the inverse or an approximate inverse of a linear periodic filter. We use the state equation description. We give necessary and sufficient conditions for the existence of the inverse, and we give a simple formula to compute it as a periodic filter with the same period. In case the inverse does not exist, i.e., in not implementable as a causal filter, we propose a method to find an approximate inverse which has a property that, when it cascades with the original periodic filter, the overall cascade connection is a pure delay of minimal possible length.

In our analysis, a single-input single-output (SISO) $N$-periodic digital filter is represented as an $N \times N$ proper rational matrix in : with strictly proper upper off-diagonal entries, as discussed in [5] and [8]. This model corresponds to the block signal processing structure [9, ch. 10]. This representation yields considerable simplification in analysis.

In Section II, we state precisely the problem under consideration and the transfer matrix representation for periodic filters. In Section III, we derive the necessary and sufficient condition for the existence of the inverse and a simple formula for computing it. A method of finding the optimal approximate inverse is proposed in Section IV. An illustrative example is given in Section V. Section VI is a brief conclusion.

Manuscript received December 14, 1991; revised February 7, 1993. This work was supported by the National Science Council of the Republic of China under Grant NSC 79-0404-E0009-07. The associate editor coordinating the review of this paper and approving it for publication was Prof. Sergio D. Cabrera.

C.-A. Lin is with the Department of Control Engineering, National ChiaoTung University, Hsinchu, Taiwan, R.O.C.

C.-W. King is with the Institute of Electronics, National Chiao-Tung University, Hsinchu, Taiwan, R.O.C.

IEEE Log Number 9213280. 